\title{
Comunicación efectiva en Lengua Extranjera a través del Aprendizaje Basado en Tareas
}

\author{
María José Gómez Perales
}

Universitat Politècnica de València, mjgomez@idm.upv.es

\begin{abstract}
Task Based Learning and Content Based Instruction approach are the methodolgical resources that nourish the present proposal. The enterprise, as transversal content, served as guide when proposing a series of tasks to the students who, with a high level of success, developed the ability of communicating effectively in German, in the context of the optional subject German B2.
\end{abstract}

Keywords: Task Based Learning, Foreign Language, German, Effective Communication

\begin{abstract}
Resumen
El Aprendizaje basado en Tareas (Task Based Learning) y el enfoque de la enseñanza de lenguas extranjeras a través de contenidos (Content-Based Instruction) son los recursos metodológicos que sustentan la presente propuesta. La empresa, como contenido transversal, ha servido de guía a la hora de proponer una serie de tareas a los estudiantes que, con un alto grado de éxito, han desarrollado la capacidad de comunicarse de forma efectiva en alemán, en el marco de la asignatura optativa transversal Alemán $B 2$.
\end{abstract}

Palabras clave: metodología, aprendizaje basado en tareas, lengua extranjera, alemán, comunicación efectiva.

\section{Introducción}

En el marco del aprendizaje de lenguas extranjeras en el ámbito universitario, la lengua alemana ocupa un lugar destacado en las preferencias del estudiantado de la Universitat Politècnica de València (a partir de ahora UPV). Así lo demuestran las cifras de alumnos que se matriculan en los diferentes niveles de esta lengua que, después del inglés, es la más demandada.

La relevancia, tanto económica como política, de Alemania en el conjunto de la Unión Europea, así como su prestigio en el ámbito de la ingeniería y la tecnología, hacen de este país un lugar de destino atractivo para los estudiantes que, no solo eligen alguna universidad alemana como destino de sus estancias ERASMUS, sino que identifican este país como el lugar en el que les gustaría desarrollar su futura profesión como ingenieros, administradores de empresas o incluso artistas. 
La asignatura Alemán B2 se oferta como asignatura optativa transversal en la UPV desde el curso 2015/2016 y el supuesto desde el que afronté su planificación partió de la idea de que la empresa, como estructura organizativa, sería con una alta probabilidad, el destino común de cualesquiera que fueran los potenciales matriculados en esta asignatura. Es decir, un futuro ingeniero industrial, agrónomo o informático trabajará en una empresa y tendrá que desenvolverse en ella. La empresa, por tanto, se convirtió en el contenido que, gracias a su carácter transversal, podía motivar a todos los alumnos. Al tomar esta decisión estamos situándonos del lado de los enfoques de enseñanza de lenguas que relegan la gramática a un lugar subsidiario en relación a los temas o contenidos que son tratados en el aula (ContentBased Instruction), que adquieren relevancia y guían el aprendizaje de la lengua extranjera (Crandall \& Tucker, 1990).

Por otra parte, la capacidad de comunicación efectiva, ya tenga lugar ésta por escrito o de forma oral, es no solo una de las competencias transversales que la UPV considera fundamentales en sus egresados, sino también, y como no podría ser de otra manera, la competencia que, según el Marco Europeo de Referencia para las Lenguas, se persigue que adquieran los aprendices de una lengua extrajera. Es importante destacar, por tanto, que a la transversalidad de nuestra asignatura, que pueden cursar estudiantes de muy diferentes Grados, se le añade el carácter transversal de la competencia comunicativa en general, que se vería concretada en nuestro caso en competencia comunicativa en alemán.

Teniendo en cuenta estos aspectos, la distribución de los créditos de la asignatura en "Teoría de Aula" y "Prácticas informáticas" se ofrecía como una oportunidad para planificar las sesiones prácticas como el eje sobre el que hacer girar el resto de los créditos, esto es, del tiempo disponible para alcanzar, en la medida de lo posible, el objetivo fundamental de la comunicación efectiva. Para ello estructuré las cuatro sesiones prácticas de las que constaba la asignatura desde los supuestos del Aprendizaje Basado en Tareas (Ellis 2003), ya que gracias a esta orientación metodológica se consigue trasladar el foco del proceso de aprendizaje del docente al discente. No obstante, hay que precisar, que estas cuatro tareas, que detallaremos más adelante, tendrían el nexo común de la empresa. Investigaciones recientes han demostrado la idoneidad de la aplicación del enfoque del Aprendizaje Basado en Tareas para la adquisición del alemán como lengua extranjera (Bosch, 2012 y 2017), si bien en un contexto más restringido que el nuestro, ya que en esas contribuciones los alumnos estudiaban todos un mismo Grado en Turismo, por lo que las tareas y las actividades conducentes a ellas estaban relacionadas con las necesidades comunicativas propias de esa actividad profesional.

\section{Objetivos}

El objetivo general de la presente propuesta consiste en presentar el desarrollo de actividades que integran habilidades receptivas y productivas en lengua extranjera - en el sentido de Input Processing (VanPatten, 2010)- conducentes a la elaboración de tareas por parte de los discentes, en las que se pretende alcanzar un nivel óptimo de comunicación efectiva. 
Atendiendo a los objetivos marcados en el Marco Europeo de Referencia para el aprendizaje y enseñanza de lenguas, presentamos actividades en las que los estudiantes han de escuchar y comprender exposiciones orales sobre temas académicos o profesionales que les son relevantes. Asimismo han de ser capaces de leer y comprender artículos e informes sobre temas que académica o profesionalmente son significativos para ellos. Por último, se pretende lograr que sean capaces de comunicarse, ya sea oral o por escrito, de forma eficiente sobre temas profesionales o académicos.

\section{Desarrollo de la Innovación}

Como hemos explicado en la introducción, nuestra propuesta consiste en cuatro tareas -a realizar por los estudiantes- que, de una u otra forma tienen, desde el punto de vista del contenido, el nexo común de la empresa. Las enumeramos a continuación, para pasar después a explicar con detalle cómo se han puesto en práctica cada una de ellas:

- $\quad$ Presentación oral de una empresa (Firmenpräsentation)

- Puesta en escena de una entrevista de trabajo (Vorstellungsgespräch)

- Video-Tutorial (Video-Anleitungen)

- Diálogos conflictivos en el puesto de trabajo (Konfliktgespräche am Arbeitsplatz)

En la primera tarea, el alumno ha de realizar una exposición oral (naturalmente en alemán) de una empresa, que él mismo ha elegido. Previamente, el estudiante ha elaborado una presentación en cualquier formato (habitualmente se elige el soporte Power Point). La gran mayoría de los alumnos optan por buscar información sobre una compañía relacionada con su posible futuro profesional. Esta presentación ha de ser expuesta oralmente al resto de la clase. Las actividades que se han llevado a cabo en el aula -lo que se conoce como pretareahan estado enfocadas a facilitar la elaboración y presentación de la empresa. Con ese objetivo, el profesor planifica actividades de comprensión oral y escrita relacionadas con esta temática, en las que a través de textos, escritos u orales, el alumno recibe un determinado input de vocabulario y estructuras gramaticales relevantes a la hora de ofrecer los datos de cualquier empresa. Por ejemplo, en lo que atañe al vocabulario, el tipo legal de empresa, es decir, si se trata de una sociedad anónima o limitada $(A G / G m b H)$; año de fundación de la empresa (Gründungsjahr); ciudad en la que está ubicada su sede central (Hauptsitz); número de trabajadores (Mitarbeiter); filiales (Tochterunternehmen), etc. En lo que respecta a las estructuras gramaticales, se hará notar en los ejemplos de presentaciones de empresas facilitadas por el profesor, por ejemplo, el uso de las adjetivaciones con la correspondiente atención a la declinación del adjetivo característica del alemán: "Wir sind ein erfolgreiches und stark expandierendes Unternehmen der Metall- und Kunststoff verarbeitenden Industrie.[...]”, "MAGNA ist ein weltweit tätiger Zulieferer der Automobilindustrie. [...]"; "Die deutsche Tochtergesellschaft in Frankfurt ist eine der bedeutendsten unserer Gruppe [...]”. (Estos ejemplos proceden del manual Präsentieren und Verhandeln. Véase bibliografía).

La segunda tarea consiste en que los alumnos, por parejas, representen una entrevista de trabajo. Previamente han elaborado este diálogo, en el que uno de los alumnos tomará el rol 
de jefe o jefa de personal (Personalleiter /In) y el otro el de candidato/a al puesto de trabajo (Bewerber/In). El puesto de trabajo al que aspira el candidato, ha sido elegido por cada pareja de alumnos de una página web auténtica ( https://www.monster.de/jobs/q-ingenieurjobs.aspx?incid=CA_FatFooter_popjob_ingenieur) en la que la oferta laboral se asemeja a la que podría interesar a los futuros egresados. Esta tarea conlleva una serie de actividades previas que el profesor ha detallado. En primer lugar, todos los estudiantes han elaborado su Curriculum Vitae en alemán (Lebenslauf), después de haber leído y analizado varios modelos de este tipo de documento durante la fase denominada Pretarea (https://www.monster.de/karriereberatung/artikel/musterlebenslauf-zum-download-30985). Asimismo, los estudiantes han de redactar la carta de solicitud (Bewerbunsbrief) para aspirar al puesto de trabajo que han seleccionado en la página web. Este tipo de documento ha sido también objeto de estudio y análisis en la fase previa a la tarea, mediante la lectura de diversos ejemplos (https://www.monster.de/karriereberatung/lebenslaufbewerbungsschreiben/anschreiben-muster). Y por último, también el diálogo típicamente característico de la entrevista de trabajo ha sido tematizado en el aula por medio de ejemplos.

La tercera tarea consiste en la grabación de un vídeo-tutorial por parte de los alumnos, en el que presenten un objeto/producto o un proceso. $\mathrm{Al}$ igual que en las tres tareas anteriores, el profesor ha diseñado una serie de actividades con las que facilitar a los estudiantes el input necesario (https://www.youtube.com/watch?v=3_IBvPsTHls) que motive la producción de un vídeo propio (output). En este caso, los ítems gramaticales que tienen un mayor protagonismo son, entre otros, el modo imperativo y la voz pasiva. Por ello, el profesor guiará la atención del alumno sobre el uso de estos dos recursos gramaticales en el contexto concreto de ese tipo de "textos" y facilitará materiales que fijen el uso correcto de ese contenido gramatical.

Por último, en la cuarta tarea, los alumnos han de representar un diálogo en el puesto de trabajo, que parte de una situación conflictiva, como por ejemplo, falta de puntualidad, incumplimiento de responsabilidades, malos entendidos, etc. Previamente, los alumnos han decidido, por parejas, cuál será el conflicto entre ambos y redactarán el diálogo con unas estrategias discursivas u otras, dependiendo de que hayan decidido que el conflicto se resuelva o no. El profesor, por su parte ha ofrecido situaciones similares por medio de actividades en el aula como trabajo previo a la tarea de los estudiantes (véase en este caso la unidad 13 "in Konfliktsituationen reagieren" del manual Im Beruf, así como esta misma unidad en el volumen de ejercicios).

\section{Resultados}

Es importante destacar que la primera y la tercera tarea- es decir, la presentación de la empresa y el tutorial- son trabajos individuales realizados por cada estudiante con el objetivo de ser presentados posteriormente al resto de la clase. Mientras que la segunda y la cuarta tareas son realizadas en grupos de dos o, en algunos casos, de tres personas. Por otra parte, hay que señalar que la presentación de la empresa y el tutorial son tareas que el

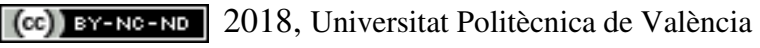

Congreso IN-RED (2018) 
estudiante ha elaborado fuera del aula; mientras que la entrevista de trabajo y el diálogo sobre una situación conflictiva son realizados en la clase. Además, se ha de destacar el hecho de que en la primera tarea el estudiante presenta su empresa en el aula, mientras que el caso del tutorial, al tratarse de un video, los estudiantes autores del trabajo, son espectadores de su propio trabajo en el aula.

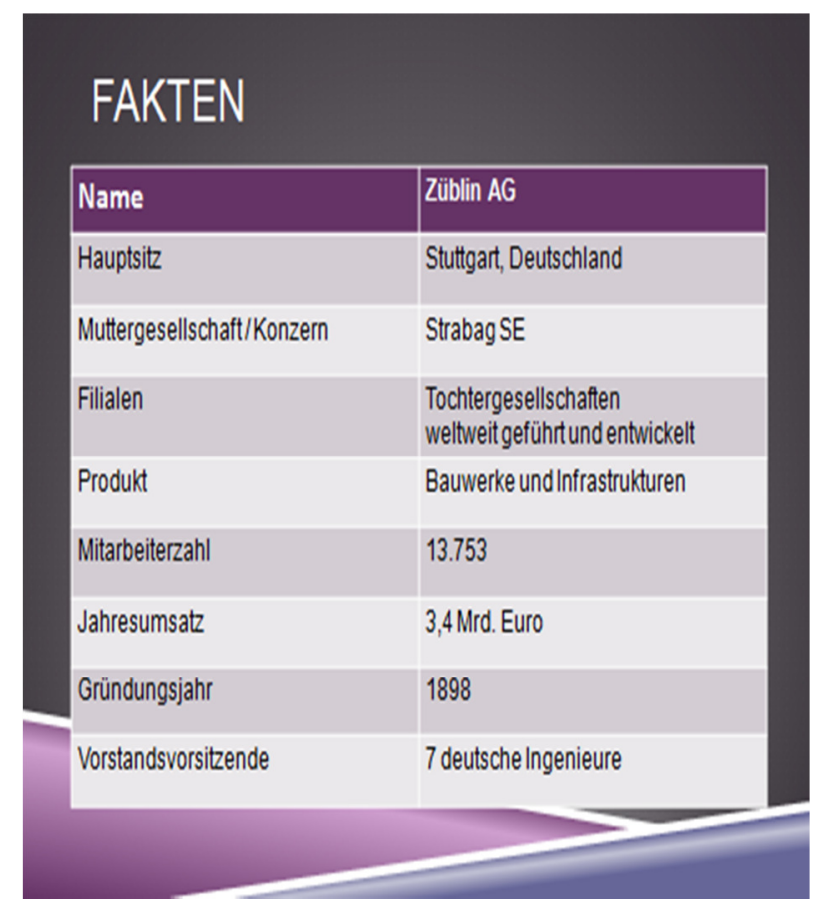

Fig. 1 Diapositiva de una las empresas

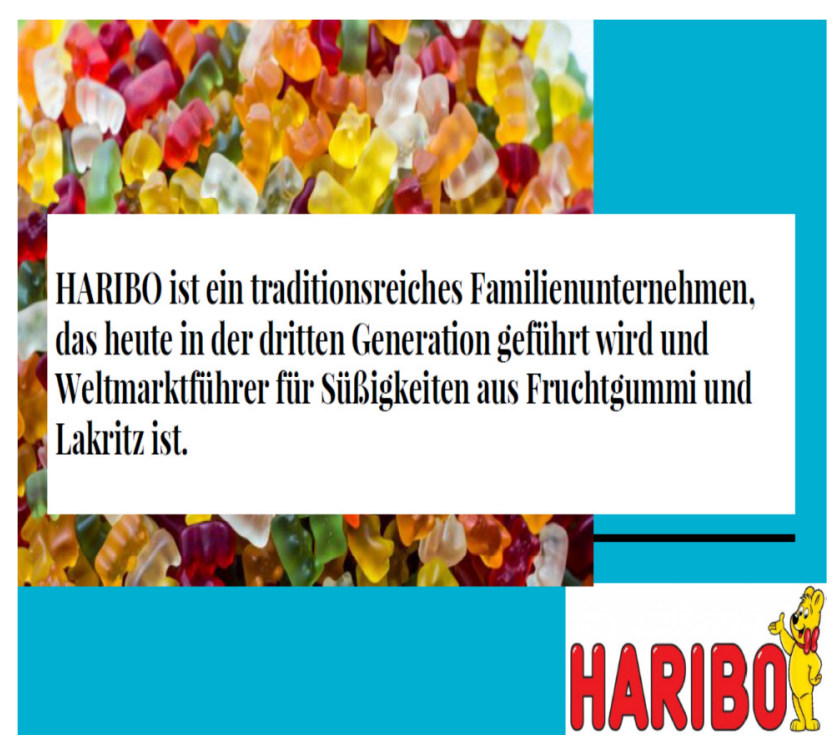

Fig.2 Diapositiva de otra de las empresas

(cc) EY-NC-ND 2018, Universitat Politècnica de València 
Los resultados de la primera tarea que presentaron los alumnos, tuvieron una extensión que osciló entre las 10 y las 15 diapositivas y las presentaciones orales una duración que, dependiendo de la fluidez y la rapidez de la dicción, se extendió entre tres y los seis minutos. Por otra parte, los vídeos tutoriales tuvieron una duración de entre tres y cinco minutos.

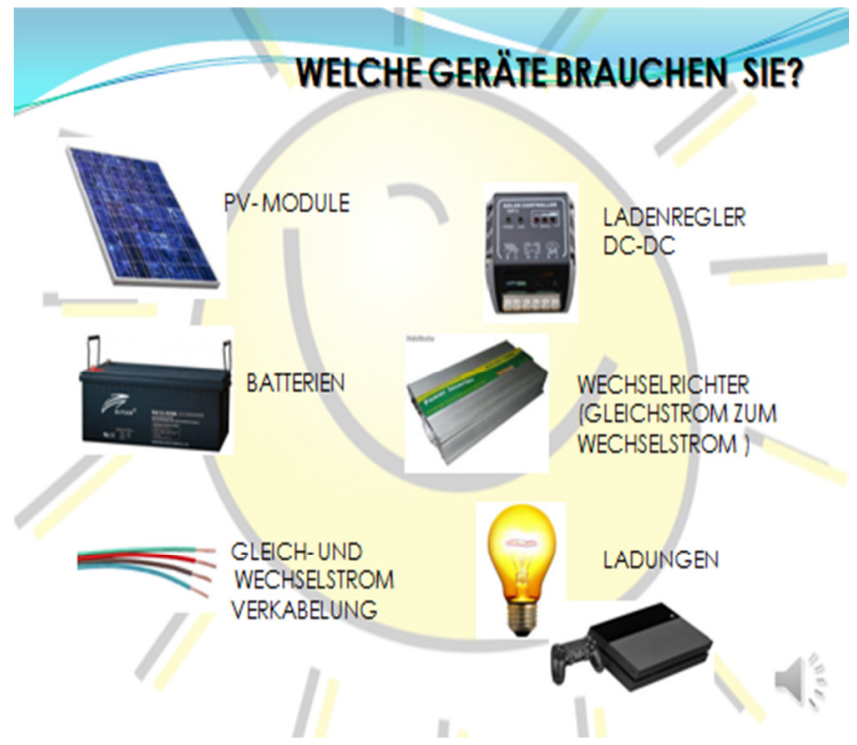

Fig. 3 Captura de una de las pantallas de un tutorial

En las siguientes direcciones de youtube pueden verse algunos de los tutoriales realizados por los estudiantes: https://www.youtube.com/watch?v=MgJG4PYbulg\&feature=youtu.be, https://www.youtube.com/watch?v=2qG-Vtc0xLk.

A continuación reproducimos un fragmento de la entrevista de trabajo redactada por un grupo de tres estudiantes. Como hemos explicado anteriormente, los alumnos han realizado un trabajo previo consistente en la búsqueda de un puesto de trabajo en una web auténtica, la elaboración del curriculum vitae y la redacción de la carta de solicitud de empleo. Todas esas actividades previas condujeron finalmente a las entrevistas de trabajo, que fueron grabadas, en este caso, como archivos de audio.

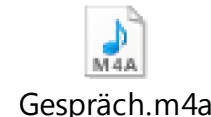

\section{*DAS VORSTELLUNGSGESPRÄCH}

P: Guten Morgen, Herr López. Mein Name ist Pablo Álvarez und sie heißt Marta Pérez. Wir freuen uns, Sie kennenzulernen.

M: Guten Mogen, wir sind für die Personalabteilung von dieser Firma zuständig. Nehmen Sie bitte Platz.

(c) EY-NC-ND 2018, Universitat Politècnica de València

Congreso IN-RED (2018) 
J: Guten Tag, ich freue mich auch, Sie kennenzulernen.

M: Haben Sie uns einfach gefunden?

J: Ich hatte Schwierigkeiten, Parkplatz zu finden aber trotzdem bin ich rechtzeitig angekommen.

P: Machen Sie sich keine Sorgen. Die Mitarbeiter haben bei uns freien Parkplatz.

J: Schön zu wissen.

M: Erzählen Sie uns bitte, wo Sie studiert haben.

J: Aus familieren Grunde habe ich in Berlin die Grundschule und das Gymnasium besucht. Dort habe ich meine Deutschkenntnisse verbessert.

P: Sie haben schon Berufserfahrung. Welche waren Ihre Aufgaben bei Dragados?

J: Dort habe ich drei Monate einen Praktikum absolviert und habe mich mit der Plannung, Herstellung und dem Betrieb von Bauwerken beschäftigt. Ich musste viel unterwegs sein.

M: Das ist aber interessant! Das passt auch zu dieser Stelle. Worin sehen Sie Ihre Stärken?

J: Ich fuhle mich gut im Team zu arbeiten und meine Kommunikationsfähigkeit mitzuteilen.[...]

Como puede observarse, hay algunos errores de ortografía (*Gründe; *fuhle, *Plannung) o de gramática, como en la flexión de los casos (*einen Praktikum), por ejemplo. No obstante, el nivel es más que aceptable y lo verdaderamente interesante desde el punto de vista didáctico, es saber que la tarea ha sido realizada por el grupo después de haber consensuado una serie de decisiones, como la de determinar quién ocuparía cada uno de los roles, así como la de decidir qué anuncio elegían de la oferta de puestos de trabajo y de apoyarse en el curriculum real de la persona que hacía las veces de candidato.

En lo que respecta a la última tarea, reproducimos también uno de los diálogos sobre una situación conflictiva en el puesto de trabajo, que fue asimismo grabado como archivo de audio:

\section{*Situation 1:}

-Herr Müller, wo ist der Jahresbericht?

+Ach ja, ich habe es schon angefangen, in zwei Wochen wird es fertig sein.

-Der Termin ist heute! Das kann doch nicht wahr sein!

+Heute? Ich habe gedacht dass es für den ersten Juli wäre.

- Ersten Juni! Das ist doch die Höhe! Wie könnten Sie den Termin vergessen?

+Es tut mir aber leid, ich hatte ...

-Das ist mir aber egal! Ich gehe jetzt zum Chef!

+ Wissen Sie was, Herr Bensalah? Dieser Bericht ist auch Ihr Job!

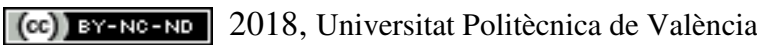
Congreso In-Red (2018) 
- Aber meinen Sie dass ich immer alles allein machen muss? Ich habe viel mehr Arbeit und Sorgen... Sie müssten nur den Bericht machen!

+ Also, das geht zu weit. Ich gehe selbst zum Chef.

$<$ Was ist hier los? Warum streitet ihr euch? Ich möchte von euch beiden nichts mehr hören! [...].

\section{Conclusiones}

Las tareas presentadas en este trabajo -realizadas por los estudiantes- muestran la complejidad que subyace en los actos comunicativos, ya sean estos presentaciones o diálogos, y también evidencian la necesidad de diseñar actividades previas -por parte del profesor- que conduzcan al éxito de la tarea. En cualquier caso, y coincidiendo con Van Dijk (2003) podemos concluir que "la teoría del contexto explica cómo los participantes son capaces de adaptar la producción y la recepción/interpretación del discurso a la situación comunicativa interpersonal-social", que en nuestro caso concreto es diferente en cada una de las tareas. Por otra parte, la relación que se establece, ya sea por parte del estudiante hacia los destinatarios de sus tareas -público que atiende a la presentación de la empresa o espectadores del vídeo tutorial- así como la relación con sus interlocutores en la elaboración y representación de los diálogos - entrevista de trabajo y diálogo conflictivoponen de manifiesto la relevancia de la interacción social como condición de aprendizaje (Suárez Guerrero, 2010).

En nuestra propuesta hemos destacado la utilidad de grabar las tareas realizadas por los estudiantes, acción que es llevada a cabo en la mayoría de los casos por ellos mismos, y que ha supuesto agregar un documento novedoso a la tarea encomendada. Los estudiantes han tenido que presentar archivos de audio o de vídeo con los resultados de sus tareas y estos documentos han tenido una doble utilidad; bien han servido para la evaluación por parte del profesor; o para la autoevaluación y reflexión propias por parte de los estudiantes.

En las encuestas de la evaluación de la docencia por parte del alumnado, la asignatura Alemán $B 2$ obtiene unos resultados bastante satisfactorios (una media de 8,75 puntos sobre 10 ), en los ítems relacionados tanto con los materiales proporcionados o recomendados (Pre-tareas); así como con aquellos relativos a la metodología empleada y las actividades realizadas. Un noventa y cinco por ciento de los estudiantes considera que las prácticas realizadas ayudan a aprender y a alcanzar un grado óptimo de competencia a la hora de comunicarse en alemán.

El desarrollo metodológico del Aprendizaje Basado en Tareas aplicado a la adquisición de la comunicación efectiva en alemán se ha revelado, por todo ello, como una herramienta útil que podría ser puesta en práctica también en el aprendizaje de otras lenguas extranjeras. 


\section{Referencias}

BOSCH ROIG, G. (2012). "Aufgabenorientierung mit proaktiver Formfokussierung als didaktisches Konzept für den Deutschunterricht im Tourismus” en IBERICA, 23, p. 157-172.

BOSCH ROIG, G. (2017). "Structured Input Activities como medio para desarrollar estrategias de Atención a la Forma (Focus on Form) en un Contexto de Aprendizaje de Alemán Basado en Tareas" en PORTA LINGUARUM, 28, P.127-140.

BUHLMANN, R., FEARNS, A., GASPARDO, N. (2003). Präsentieren und Verhandeln.Wirtschaftsdeutsch. Warschau: Poltext. Goethe Institut Internationes.

CRANDALL, J., TUCKER, R. (1990). "Content-based language instruction in second and foreign languages" en ANIVAN, S. Language teaching methodology for the nineties. Singapore: SEAMEO Regional Language Centre, p.83-96.

ELLIS, R. (2003). Task-based Language Learning and Teaching. Oxford: Oxford University Press.

HAGNER, V., SCHLÜTER, S. (2014). Im Beruf. Arbeitsbuch. Deutsch als Fremd- und Zweitsprache. $B 1+/ B 2$. Ismaning: Hueber

MÜLLER, A., SCHLÜTER, S. (2013). Im Beruf. Kursbuch. Deutsch als Fremd- und Zweitsprache. $B 1+/ B 2$. Ismaning: Hueber.

SUÁREZ GUERRERO, C. (2010). Cooperación como condición social de aprendizaje. Barcelona: Editorial UOC.

VAN DIJK, T. (2001). "Algunos principios de una teoría del contexto" en $A L E D$. Revista latinoamericana de estudios del discurso, 1(1), 2001, p. 69-81.

VANPATTEN, B. (2010). "Input Processing in SLA" en VANPATTEN, B. Processing Instruction. Theory, Research, and Commentary. NY: Routledge, p. 5-31. 